\title{
Mobile consultant: combining total mobility with constant access
}

\author{
Konstantinos A. Banitsas ${ }^{1}$, Pantelis Georgiadis ${ }^{2}$, Sapal Tachakra ${ }^{3}$, Dionisis Cavouras ${ }^{2}$ \\ ${ }^{1}$ School of Engineering and Design, E\&CE, Brunel University, West London, England \\ ${ }^{2}$ Medical Image and Signal Processing Lab, Technological Educational Institution of Athens, Greece \\ ${ }^{3}$ A\&E department, North West London Hospitals, England
}

\begin{abstract}
Minimizing the time required for a medical consultant to offer his/her expert opinion, can be viewed as a life-saving procedure. We have designed and tested an integrated system that will allow a medical consultant to freely move either within, or outside the hospital, while still maintaining constant contact with the patients via videoconferencing and high-resolution imaging. The above system is explained in this paper, along with its advantages and its potential limitations. Conclusively, we demonstrate that such a system further increases the mobility of the medical consultant, while improving the healthcare service.
\end{abstract}

\section{INTRODUCTION}

$\mathrm{T}_{\mathrm{n}}^{\mathrm{H}}$ HE nature of the medical profession usually dictates that medical doctors have to be available and on-demand, most of the time. This is especially true for medical consultants that are required to give other doctors their expert opinion. As the later are usually assigned to support a wider area (group of hospitals), it is often that they cannot be physically present next to the patient and, often, have to wander inside their hospital or even travel between hospitals.

Until now, telemedical devices have been used to bridge this gap between the distant expert and the medical personnel in need of an expert opinion. These devices are usually cumbersome, require expert installation, have high cost and usually, due to their volume, require a dedicated room to operate. The patient has to be moved to such a videoconferencing room; a procedure that is time consuming and potentially dangerous for the patient's health [1], [2].

An improvement of this situation was presented some years ago with a system called MedLAN. It was a wireless system that allowed videoconferencing, usually between the Accidents and Emergency wards (A\&E) of a hospital and another stable point where the medical consultant would reside (usually within another hospital). It was light and easily manageable and reduced the cost of communications considerably as, in contrast with its predecessors, it used wireless networks and the hospital's backbone to transfer the streaming data instead of the conventional triple-ISDN line [3]. Unfortunately, this system allowed mobility only on one side (A\&E) and assumed that the medical consultants would be in a fixed position, although some improvements of the system demonstrated mobility through GPRS [4].

There was a demand expressed by numerous doctors, for a system that would allow consultants to move freely within different wards of the hospital while being in constant contact with any doctor in need of their expertise [Fig. 1]. Furthermore, there should be a way of achieving the above not only in the hospital's vicinity, but as the consultant is roaming outdoors, commuting, residing in his/her house, etc. The whole system had to be efficient enough to allow for safe diagnostic evaluation, light enough so the consultant can carry it anywhere, cheap enough so the hospital administration could support the idea of using it and simple enough so it can be used with the minimum amount of training [5].

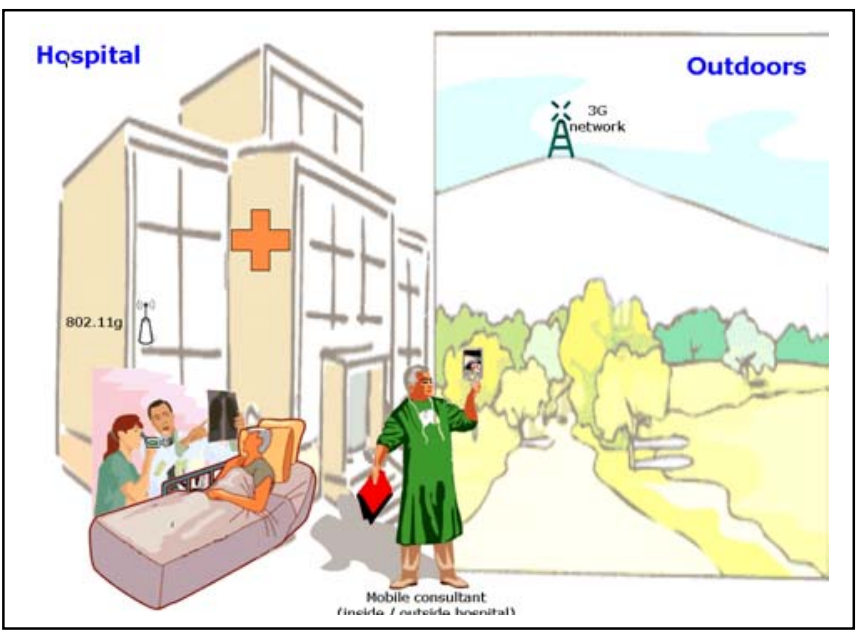

Fig. 1. A medical consultant is allowed to freely move inside the hospital (IEEE $802.11 \mathrm{~g}$ ) as well as outdoors (3G) while videoconferencing with the treating doctors, using just a light PDA.

The implementation of such systems seemed impossible just a few years ago. The hardware was relatively big the computational power was limited and the communication speed was slow [6]. Recent advances, however, introduced handheld systems that are much lighter and practically emulate the abilities of an ordinary computer (high processing power, substantial memory, high screen resolution, wireless network capability), while at the same time can act as a third generation (3G) mobile phone and support videocalling. It is on such a system that the current research was based.

\section{Methodology}

The research was divided into three stages: initially, the most suitable hardware was selected among several 
alternatives. Following, the videoconferencing software and the image viewer was developed and finally an initial clinical test was carried out in order for the medical consultants to evaluate the performance of the system.

\section{A. Hardware}

It was clear that in order to cover both the indoor and outdoor environment, a device that had the capability of operating both as a PDA (or personal laptop) and a $3 \mathrm{G}$ mobile phone, had to be selected. An excellent candidate was the HTC Universal PDA (also sold by other companies bearing the names Qtek 900, JasJar, i-mate). It incorporated numerous desirable characteristics: had a CPU running at $520 \mathrm{MHz}$, a flash memory up to $4 \mathrm{~GB}$, a crystal clear, highresolution screen capable of displaying 640x480 pixels, wireless network access, bluetooth support and was running Windows Mobile 2005; an operating system that made it compatible with any other similar device and with an easily available Software Development Kit that allowed for applications to be developed. Furthermore, the device supported mobile telephony in four bands (three GSM and UMTS) thus allowing for videoconferencing on the go. The device also included an embedded keyboard for data entry, Internet explorer, Outlook email, Word, Excel, PowerPoint and many other applications. The overall weight of the PDA was just under $290 \mathrm{~g}$, which made it very easy to be carried anywhere, even inside a pocket.

Network access could be established in a number of ways but by default it started with IEEE $802.11 \mathrm{~b}(11 \mathrm{Mbps})$ and whenever the device could not find an access point, it reverted to a $3 \mathrm{G}$ connection (UMTS) through the mobile network and with a maximum speed of $384 \mathrm{Kbps}$ [7].

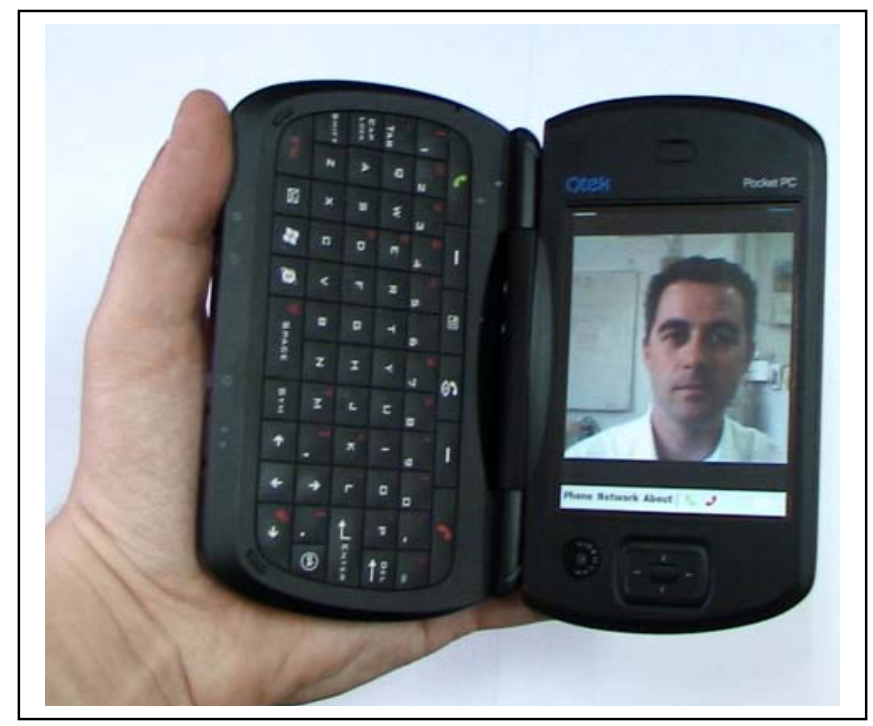

Fig. 2. Mobile videoconferencing while within a hospital: the medical consultant only has to carry a light PDA.

\section{B. Software}

Two distinct pieces of software were developed by the authors, at the Medical Image lab of TEI Athens.

The VoIP module handles the video and audio transfer between the (wired or wireless) system that resides in the place in need of an expert opinion (e.g. A\&E ward) and the mobile consultant. The system was deliberately developed to offer one-way video and two-way audio (the patient's side was not in need of viewing the consultant) thus limiting collisions and minimizing the bandwidth demanded.

In the patient's side, any software capable of sending video and audio through the H.323 protocol would be able to communicate with the PDA unit. Microsoft NetMeeting was used in the initial experiments and performed adequately. It is included free of charge in all Windows suits and can be set to contact the remote node in a number of ways; the most practical was calling on its IP number. After a delay of about 1-2 seconds, the consultant's PDA will ring; the user will accept the call and the videoconferencing procedure will be initiated [Fig. 2].

Apart from the video (that sometimes might have limited quality due to the specifics of the H.261 algorithm), it is also extremely useful for the treating side to be able to send highquality images to the medical consultant. These might include x-rays, CT images, MRI, ultrasound scans, etc. In contrast with the video that highly compresses the datastream to preserve bandwidth and reduce frame rate fluctuation, images can be sent intact for the consultant to study and evaluate.

PDAImaging is an image viewer that is tailor made for medical applications. It includes operations like pan, zoom in/out, ROI, and most importantly, several filters (windowing, histogram modification filters, convolution filtering) that will assist the doctor in his/her diagnosis. It also supports storing the received images locally; even in DICOM format. It can run alongside the VoIP module so the images can be transferred at the same time as the wireless videoconferencing takes place.

\section{Clinical testing}

A prototype of the system was developed at the beginning of the year and was initially tested in the Central Middlesex Hospital in West London. Despite its thick old walls, the environment of this hospital was "wireless friendly" as, not so long ago a wireless medical project was implemented there [8]. The system was tested for indoor operation in the Accidents and Emergency ward (A\&E) of the hospital. This area was covered by five Access Points (APs) that were configured so that their transmissions will not interfere with each other (in IEEE $802.11 \mathrm{~b} / \mathrm{g}$ there only three independent frequencies. As a result, one should strategically place the APs so that the ones using the same frequency are far apart from each other). 
The three consultants that tested the system were given the device and were told to try and use it in two scenarios: videoconference inside the hospital using the internal wireless network and also, use it outdoors while connected to $3 \mathrm{G}$ network. There were fourteen sessions overall where several videos were sent (more than half were prerecorded in order to minimize the nuisance to the patients) along with a number of medical images (x-ray, CT, MRI, US) [9]. Three of these sessions were tested while outside the hospital. Consultants were asked to evaluate both videos and still images in terms of: video clarity, frame rate, diagnostic ability, image resolution, image clarity and overall diagnostic ability for the purpose of making a safe distant diagnosis.

\section{RESULtS}

The overall results were very encouraging and demonstrated that the system can be used on a larger scale.

Videoconferencing and image transfer should be evaluated in two modes: using the hospital's internal wireless network and using the mobile provider's 3G network.

The former behaved more or less as expected. The PDA was capable of videoconferencing in a number of resolutions ranging between $176 \times 144$ and $352 \times 288$ pixels. Although the necessary bandwidth for the highest resolution was available without any major dropping of the frame rate, it was rarely used, as it will impose an additional load to the wireless network that might prevent other people accessing it. The frame rate varied between 5 and 15 frames per second, depending on the distance between the mobile user and its associated AP (802.11 connection speed falls back to 5.5, 2 and $1 \mathrm{Mbps}$ as the signal deteriorates). When the movement of the source camera increased, the image on the receiving end appeared relatively "squared" but this is to do with the way that the compression algorithm behaves [10].

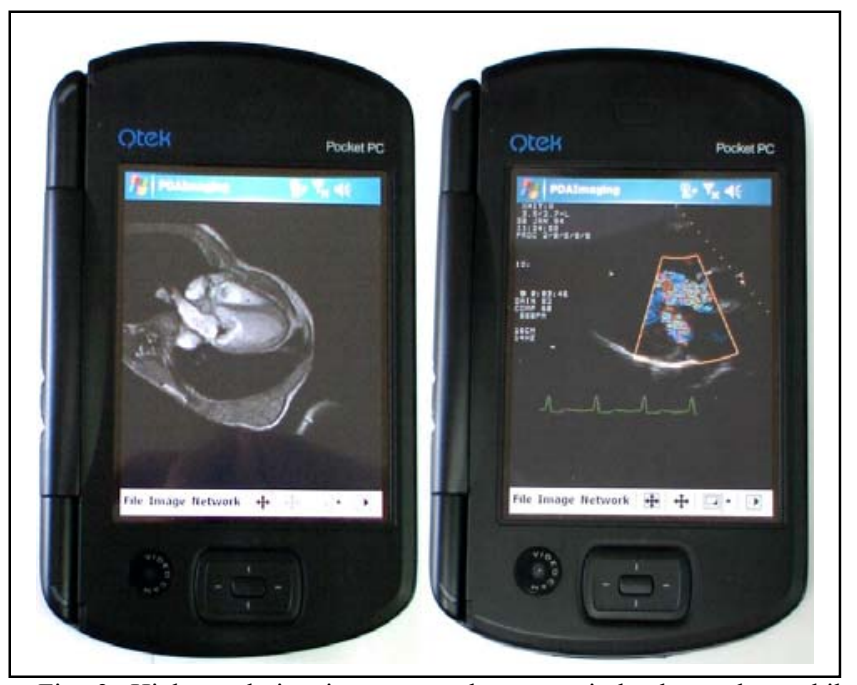

Fig. 3. High resolution images can be sent wirelessly to the mobile consultant, either within or outside the hospitals vicinity.
Sound was relatively clear (better than telephone conversation) with a source sampling rate of $8 \mathrm{KHz}$ and resolution of 8 bits per sample. Apart from the PDA's stereo speakers, the user had the ability to connect to a hands-free so both the incoming and the outgoing sound will sound clearer.

High-resolution images of various sizes could be wirelessly sent to the mobile unit. Zooming and panning was easily performed using either the PDA's pen or one's finger. The average time for a $500 \mathrm{~KB}$ image to be sent over to the consultant varied between 2-5 seconds.

In the case that the consultant was away from the hospital, he/she could also gain access through the ever-increasing number of hot-spots around urban centers.

However, if this fails, the device is ready to engage in a standard videoconferencing through normal video calling. This allows for a relatively low frame rate (3-5 fps) and low resolution (in comparison with accessing the wireless network), as it only uses $64 \mathrm{Kbps}$ of streaming data. The advantage, however of having access from anywhere there is UMTS coverage, overcomes the limitations [11].

Alternatively, one can use a prepaid "data packet" by the mobile provider and achieve speeds up to $384 \mathrm{Kbps}$ downstream so one can, more or less, enjoy the same video quality as when wireless LAN was used [7].

It is worth mentioning that there is nothing that would prevent seamless transition from one network to another: as the mobile consultant exits the hospital, the PDA will automatically reconnect to the $3 \mathrm{G}$ network without loosing the communication link. However, this introduces an average of a 20 second gap and also assumes that the hospital's firewall would be open to accept the incoming connection by an outside provider; namely the mobile company.

Security of the system in the case of WLAN resides on the use of WEP, EAP or MAC filtering, while in the mobile provider scenario it is left in the proper use of the standard GSM/UMTS security and encryption measures.

Another point to consider is the ability of the system to act as a remote / mobile terminal of the hospital network: the mobile PDA can access the network, load, save, print, check emails and use the Internet as easily as using a desktop computer (the use of its internal keyboard makes it even easier). This is an advantage in a place such as a hospital, where space is limited and cables running in the floor can pose a threat to the fast moving healthcare personnel.

It can also be used as a form of beeper that is capable of sending simple messages and furthermore as a cordless phone so any doctor / consultant can be reachable anywhere, anytime and at no cost to the hospital (as long as he/she is in the WLAN range).

\section{DISCUSSION}

The prototype system behaved satisfactory in the initial 
test phase. Apart from some "freeze-up" of the video during videoconferencing (mainly attributed to the access on the wireless network) both video and sound were of good quality.

Doctors that tested the system agreed that it would be beneficial to their profession, as it will allow them to be more accessible while performing other duties. They also agreed that the outputs of the system were of acceptable diagnostic quality $(80 \%)$ and in most of the cases allowed for a safe diagnosis and consultation (75\%).

The cost of the hardware (assuming that a wireless LAN installation already exist in the hospital) is about 1000 Euros per unit but is likely to go down with time. Videoconferencing within the hospital is completely free while the airtime for videoconferencing outside the hospital's WLAN range is about 10-20 Euros per 5-minute session (depending on the mobile provider).

Although the system has increased interoperability (follows standard videoconferencing algorithms, can read / save in DICOM format, uses IEEE $802.11 \mathrm{~b} / \mathrm{g}$ networks), it is mostly designed as a stand-alone, independent piece that would not rely on the existence of any other system in order to operate.

Overall, what the researchers of this system envision, is that not only the medical consultants but all the medical personnel in a hospital will gradually replace their beepers, mobile phones and videoconferencing devices, by such an all-in-one system that is light enough to be carried anywhere.

\section{CONCLUSION}

In this paper, we presented an integrated system that will, among other services, allow mobile and wireless videoconferencing between a roaming consultant and a base station (usually in a hospital). The system behaves satisfactory in most conditions and provides the medical consultant with a number of desirable characteristics. Overall, it minimizes the time for a doctor to get expert consultation and thus improves the quality of care for the patient.

\section{REFERENCES}

[1] A. C. Norris, "Essentials of Telemedicine and Telecare", John Wiley \& Sons Ltd, 2002

[2] Hjelm M., Wootton R and Craig J. "Benefits and drawbacks of telemedicine, Introduction to Telemedicine", Royal Society of Medicine, London, 1999, Chapter

[3] Konstantinos A. Banitsas, et. al., "Applications of Medical Wireless LAN Systems (MedLAN)", International Journal for Medical Marketing, vol. 2, no. 2, pp. 136-142, Jan 2002

[4] Konstantinos A. Banitsas, et. al., "Using handheld devices for realtime wireless Teleconsultation", IEEE EMBC 2004, San Francisco, pp. 3105-3108, Sep 2004
[5] Ying-Wen Bai; Yung-Sung Huang, "Design and implementation of a portable medical streaming media system Enterprise networking and Computing in Healthcare Industry", 2005. HEALTHCOM 2005. Proceedings of 7th International Workshop on 23-25 June 2005 Page(s):25 - 30

[6] Pavlopoulos, S, et. al: "A novel emergency telemedicine system based on wireless communication technology - AMBULANCE", Information Technology in Biomedicine, IEEE Transactions on, Vol. 2 , Issue: 4 , pp. 261-267, Dec. 1998.

[7] Konstantinos A. Banitsas, et. al., "Using 3G links to develop a teleconsultation system between a moving ambulance and an A\&E base station", International Journal of Telemedicine and Telecare, vol. 12, p. 23-25, Royal Society of Medicine press, 2006

[8] Banitsas A. Konstantinos, Sapal Tachakra, Robert S. H. Istepanian, "Operational Parameters of a Medical Wireless LAN: Security, Range and Interference issues", Presented at IEEE EMBS conference 2002, vol. 3, pp. 1889-1890, Oct 2002, Houston

[9] Della Mea V, Wootton R and Craig J. "Pre-recorded telemedicine", Introduction to Telemedicine, Royal Society of Medicine, London, 1999, Chapter 3

[10] lain E. G. Richardson, "Video Codec Design Developing Image and Video Compression Systems", John Wiley \& Sons, Ltd, 2002

[11] Romero J., Melero J. "GSM, GPRS and EDGE Performance: Evolution Towards 3G/UMTS", John Wiley \& Son, 2003 